\title{
Deep Learning and IoT to Assist Multimorbidity Home Based Healthcare
}

\section{Césa Fonseca ${ }^{1 *}$, David Mendes², Manuel Lopes ${ }^{3}$, Artur Romão ${ }^{4}$ and Pedro Parreira}

${ }^{1}$ Departamento de Pneumologia, Universidade de Évora, Portugal

${ }^{2}$ David Mendes, DECSIS, Universidade de Evora, Portugal

${ }^{3}$ Manuel Lopes, PhD, Departamento de Enfermagem, Universidade de Évora, Portugal

${ }^{4}$ Artur Romão, MsD,DECSIS, Innovation and Development, Portugal

${ }^{5}$ Pedro Parreira, PhD, Department of Nursing and Management, Nursing School of coimbra, Portugal

\begin{abstract}
The authors present a proposal to develop intelligent assisted living environments for home based healthcare in the presence of multimorbidity chronic patients. These environments unite the chronicle patient clinical history sematic representation ICP (Individual Care Process) with the ability of monitoring the living conditions using loT technologies and events recurring to a fully managed Semantic Web of Things (SWoT) and Machine Learning Algorithms in order to activate the LDC (Less Differentiated Caregiver) for a specific care need. With these capabilities at hand, home based healthcare providing becomes a viable possibility reducing the institutionalization needs. The resulting integrated healthcare framework will provide significant savings while improving the generality of health and satisfaction indicators.
\end{abstract}

Keywords: Computer reasoning; Knowledge representation; Deep learning, IoT; WBAN

\section{Introduction}

There is a major need for ICT systems to assist the caregivers who are attending patients suffering from chronic conditions. In the middle of the last century, countries designed and implemented health systems for attending acute health problems, but the demographic changes that the world has witnessed in the last decades mandate different responses geared towards home and community based care where multimorbidity is a constant.

We join the capabilities of having computable extended health/ disease process knowledge, that we call ICP (Individual Care Process), enriched by IoT enabled ambient assisted living to acquire and process large numbers of health related events and automatically reason upon them. We look at the several questions that need we need to address when applying the IoT and SWoT (Semantic Web of Things) in the healthcare process and the public system. Regarding the Clinical KR, we found that the problem known as "Knowledge Acquisition Bottleneck" [1] is currently the major obstacle for the development of adequate representations of medical knowledge computable representations, namely ontologies in the specific domain and in particular in the healthcare sub domain.

We extend the usual DRS (Discourse Representation Structure) that usually handles single texts to our proposal based in a framework of ontologies ranging from the multimorbidity clinical facets to the nursing care process, which has the expanded capability of representing the whole discourse of a patient's healthcare history.

We develop the issue addressed in this paper that is the utilization of the IoT related technologies in Ambient Assisted Living, with all the underlying questions and possible problems that have to be circumvented when multimorbidity is present and propose ways of pragmatically handle them when needed and possible. Finally in the last section conclusions are drawn.

\section{The ICP (Individual Care Process)}

In any specific scientific domain the ontology creation and development has traditionally been in charge of multidisciplinary domain experts that have to master both their particular scientific field and the ontology engineering novel techniques. We introduced in the way to acquire generic medical knowledge. Shifting to the healthcare process KR (Knowledge Representation) [1], based in developments made under the auspices of 4IE (Instituto Internacional de Investigação e Inovação do Envelhecimento) a project that involves several academic and healthcare institutions from Portugal and Spain, we implemented the model called IPC.

\section{The ontological framework for the nursing process}

To be able to use the reasoning techniques and methodologies that represent the state-of-the-art in the Artificial Intelligence domain we made a thorough inventory of biomedical ontologies currently available in OWL2 format. It was found that no solution for complete Clinical Practice representation is available with enough expressiveness in its foundation for exact clinical reasoning, and we introduced the OGCP presented in the next section [2]. Formal ontologies make the semantics of terms and relations explicit such that automated reasoning can be used to verify the consistency of knowledge. To express the richness of medical knowledge without any loss of intending meaning, a broad set of ontological relations, only expressible in OWL2 using role-chains, for instance, have to be part of our TBox and it will have a SHOIN DL. While this level of representational capability is compulsory for clinical reasoning there is also an important constraint relating to the usability of our ontology that is the need to have a near real-time classification complexity because it has to be very responsive in the interactive sessions that the doctors will use when using natural language questioning. We have to use pragmatic solutions to these real constraints and OGCP is formed of a dual capability because the ABox only possesses EL++DL expressiveness. We use the ELK [3] consequence based reasoned that has polynomial time response to classify on-the-fly the CNL assertions introduced interactively by the users.

${ }^{*}$ Corresponding author: César Fonseca, Doctoral Student in Nursing, Researcher Nursing Research and Development Unit, University of Lisbon, Lisbon, Portugal, Tel: 70013046851; E-mail: cesar.j.fonseca@gmail.com

Received May 31, 2017; Accepted June 21, 2017; Published June 25, 2017

Citation: Fonseca C, Mendes D, Lopes M, Romão A, Parreira P (2017) Deep Learning and loT to Assist Multimorbidity Home Based Healthcare. J Health Med Informat 8: 273. doi: 10.4172/2157-7420.1000273

Copyright: () 2017 Fonseca C, et al. This is an open-access article distributed under the terms of the Creative Commons Attribution License, which permits unrestricted use, distribution, and reproduction in any medium, provided the original author and source are credited. 
Moreover, we accept as evidence that natural language is the Lingua Franca for communication among physicians and care providers, and we have to gather information in computable form from text that they traditionally use to pass their information. Thus, we can confirm the scope of our work in:

1. Develop Ontology suitable to model the healthcare processes accurately.

2. Develop a process for automatic acquisition of the ontology ABox from text.

3. Use this framework as support for deep learning based in IoT events and natural language CQA to use as an AI professional aid.

\section{Tutoring Process}

The first step in developing the automatic acquisition is a preparation process where the system is taught using some texts on how to properly identify and classify all the different segments. This preliminary phase needs to be done for every clinical reality that is to be subjected afterward of $\mathrm{KB}$ creation, for a specific doctor, a given service, a period or anything intended. For this preliminary step to be accomplished, we took inspiration for the supervised training in the development of a GS (Golden Standard) clinical annotated corpus presented in Roberts et al. [4]. In fact, the set of the resulting TM obtained is a GS for the accurate clinical term identification.

The learning process is based on the refinement of the Translation Memory (ies) (TM) applied to that specific acquisition. It can be seen as a "manual translation tutoring" although the focal point is not really the translation from different "natural languages" to English but, in fact, from the original controlled technical jargon to "specialized English". After the tutoring incremental process, the process for the correct Ontology Driven (OD) annotation adequately tags the found acronyms, named entities, and clinical terms taking into good account the possible event relations incurred by the specific co-occurrence of morbidities.

\section{Machine learning processes supporting the caregiver}

With the environment ready for computer reasoning, we apply ML (Machine Learning) algorithms, techniques and machinery already present in the market to implement the reasoning and LDC activation explained in the section about activation ruling ahead.

The technique used in our proposal is commonly known as supervised or predictive machine learning. In this case, the systems are instructed to learn mappings from inputs $\mathrm{x}$ to outputs $\mathrm{y}$, given a labelled set of input-output pairs called the training set. The training set in our case is the ontological A-Box guaranteed to be a GS from which the subsequent events arising from the IoT AAL are classified and handled.

\section{IoT to the rescue for intelligent life event acquisition}

Using the IoT in the healthcare process: The Internet of Things (IoT) is finding its most promising field of application in the development of AAL tools [5]. It is becoming gradually evident that the assistance needs for older adults have to rely upon automatization technologies to assist traditional human caregivers. These emerging widespread technologies available in "Smart Homes" can in fact enable less differentiated caregivers to provide assisted help. Hetero caregivers (formal and informal), if supported by technology can deliver better assistance if duly directed by evidence based AI tooling. This capability is especially evident in the vulgar case of multi-morbidity which is the most common situation amongst aging adults. The interactions of diverse drugs, therapies and other caregiving details that arise when co-morbidities are present can be handled in an integrated form if a computer based Intelligent Healthcare Supervisor is monitoring and acting in the living environment. This assisted living environment comprises a multi-tier area network consisting of Body Area Network (BAN), the LAN and ultimately the WAN. In our case we suggest the usage of a highly secure hybrid cloud for the Docker/Container based virtualization to provide the needed distributed persistence where the reasoning is to be based upon. The reasoning capabilities, tightly related to the scope of the clinical knowledge (automatically) reflected upon, range from very severe alarms that trigger rules in the BAN like a heart failure, for instance, to evidence based reasoning available in a supercomputing framework that can reason over the ICP and living conditions acquired that trigger highly sophisticated caregiving suggestions.

\section{Event acquisition in multimorbidity assisted living environments}

As seen in the previous section, our proposed systems possess the capability of reasoning about the health/disease process of a person due to the representation acquired. This clinical history can be reasoned upon and continuously enhanced through the monitoring of the daily events and activities. To implement a controlled living environment, the usual approach is to augment a home with various types of sensors and actuators. These Smart Homes gather knowledge in order to provide comfort for the residents but can be used as well to assess the cognitive and physical health of those residents. Both the living conditions and the bodily signs and symptoms need to be controlled.

\section{Sensor enabling the person and the environment}

Sensors are tending to be non-intrusive wearable and mobile. Most prominent emerging technologies range from MEMS to epidermal electronics that enable new health related sensors. Non-invasive sensors in form of patches, small holter-type and body worn devices as well as smart garments monitor health signals. Blood glucose and pressure as well as cardiac activity can be measured using wearable's that use infrared, optical and oscillometric sensing. A thorough comparison table that summarizes typical wearable and mobile sensors can be found in Memon et al. [6].

WBANs enable wireless communication around a human body. Several technologies have been proposed to achieve energy efficient communication including $[7,8]$. More recently, using the body tissue as transmission medium is being proposed. From the foundational thesis by Wegmüller [9] to the more recent works like $[10,11]$.

The amount and quality of continuous monitoring data is getting to paramount levels to what is duly considered Big Data and has to be handled as such. The huge data gathering can be pictured as an upward flow from the BAN to the WAN according to the architecture suggested in the beginning of this section. Caregiving rules activation represents the opposite flow of data/information/knowledge in a downward direction (Figure 1).

\section{Healthcare process activation ruling}

For the upward flow of knowledge, a minimal set of information is collected to generate axioms in the Functionality Ontology (OF) developed to model the WHO International Classification of Functionality (ICF) [12]. This information set on the inabilities to accomplish everyday living chores generates, when verified in relation with the healthcare process knowledge, the latest scientific evidence and the actual case based living conditions, the advising for the least differentiated caregiver. As an example we usually use the simple reminder of an automatic phone call to the informal caregiver that some medication has to be taken at a certain hour. Also a record of the therapeutic adherence is maintained in the extended healthcare process in an effort to strengthen this adherence in the aged population. 
Citation: Fonseca C, Mendes D, Lopes M, Romão A, Parreira P (2017) Deep Learning and loT to Assist Multimorbidity Home Based Healthcare. J Health Med Informat 8: 273. doi: 10.4172/2157-7420.1000273

Page 3 of 4

\section{From Smart Homes to Intelligent Homes}

Apart from providing the connected reality to the physical world of the old person, the Smart Home is now able to provide counselling and support for the healthcare activities that have to be part of the daily lives of the elder. This is made through natural interfaces that expand the Smart Phone personalities into a truly health assistant. Different issues and obstacles to ICT adherence for elder people are addressed in literature from preliminary works [13], going through important developments $[14,15]$. Policy conforming documents in the European reality [16-22]. In our work developments we take in consideration the myriad of issues when developing natural interfaces directed to the diverse caregivers ranging from the auto-care to specialized professional teams. The domiciliation of care renders huge savings as has been already demonstrated [23] while, simultaneously, improves the perceived quality of care and raises globally the health indicators. This is all possible by Healthcare Intelligent Homes that include the edge computing part of a reasoning framework that can contribute to improve the quality of the chronically ill society that we live in.

\section{AI/IoT/Big Data/Cloud frameworks for IPC reasoning}

Instead of developing a Cloud computing framework for distributed reasoning, we reviewed the market offering in the late months of 2016 regarding the overlapping concepts, weighting them and found that comparatively the most attractive environments are shown in Table 1.

The platforms where compared weighting for open vs closed source, proprietary vs public, availability of developing tools, usage pricing, semantic layer, AI APIs, Visualization tools, IoT Support, Interoperability APIs, Security and authorization APIs.

The result is that there is interest in using a mix-and-match of the diverse platforms for different usages when geared toward a specific context but mainly we are interested in Open, Java based platforms with strong developments towards IoT integration and data security embedded like FIWARE, Google Cloud, LUMADA and Universal.

\section{Conclusion}

We presented our proposal for an extended Discourse Representation Structure, the ICP that relies on extensive, and very solid, foundations like SNOMED-CT, FMA, and a Nursing Ontology among other OBO Foundry ontologies. We introduced natural language clinical reasoning aids based on distributed machine learning techniques and the options taken to implement them. We also showed how to implement these innovative AI advances and techniques in commodity hardware. Logical inferencing and clinical facts entailment that are possible through this novel capability is an interesting contribution to the application of AI to healthcare. With these tools at hand, we explore the field of Intelligent Ambient Assisted Living fuelled by the IoT and raise the veil in our proposal for home based care that can contribute significantly to a new wave of caregiving facilities that provide better quality of life for our population with co-morbidities, while keeping costs restrained and controlled.

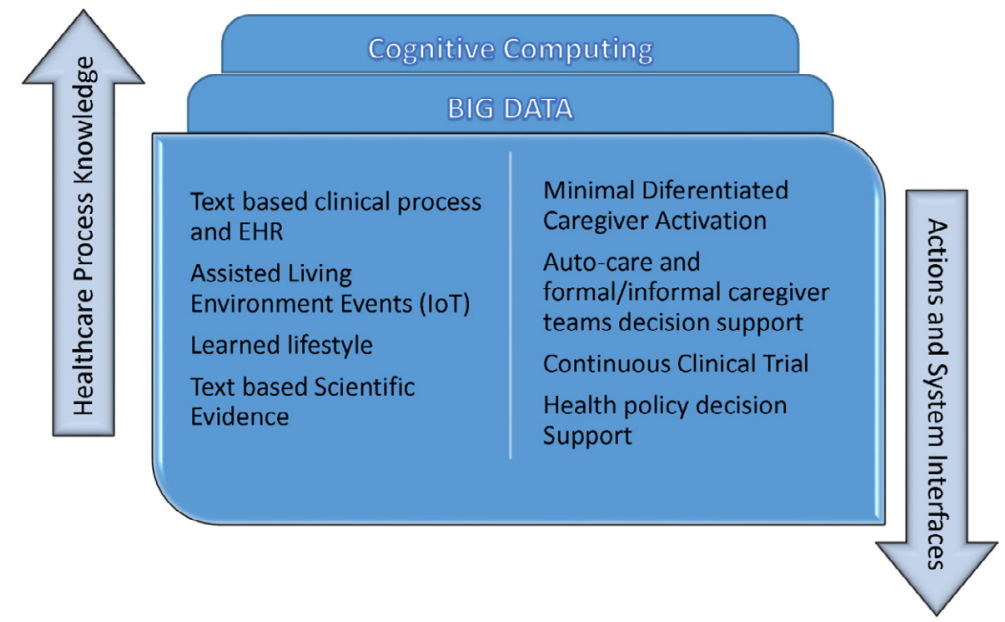

Figure 1: Knowledge flow in intelligent assisted living.

\begin{tabular}{|c|c|c|c|c|}
\hline Name & URL & Open Source & Owner & System \\
\hline AWS & https://aws.amazon.com/ & No & Amazon & Virtualization \\
\hline AZURE & https://azure.microsoft.com/ & No & Microsoft & Windows Server \\
\hline Bluemix & https://www.ibm.com/cloud-computing/bluemix/ & No & IBM & Virtualization \\
\hline FIWARE & https://www.fiware.org/ & Yes & FIWARE & OpenStack \\
\hline Google Cloud & https://cloud.google.com/ & Yes & Google & Virtualization \\
\hline LUMADA & https://www.hitachiinsightgroup.com/en-us/lumada.html & No & Hitachi & Virtualization \\
\hline OpenloT & http://www.openiot.eu/ & Yes & None & Ubuntu/Others \\
\hline Openshift & https://www.openshift.com/ & No & Ubuntu & OpenStack \\
\hline Sofia2 & https://sofia2.com/ & Yes & None & OpenStack \\
\hline UniversAAL & https://github.com/universAAL & Yes & None & Apache \\
\hline
\end{tabular}

Table 1: The market offer. 
Citation: Fonseca C, Mendes D, Lopes M, Romão A, Parreira P (2017) Deep Learning and loT to Assist Multimorbidity Home Based Healthcare. J Health Med Informat 8: 273. doi: 10.4172/2157-7420.1000273

\section{References}

1. Wong W, Liu W, Bennamoun M (2012) Ontology learning from text: A look back and into the future. ACM Comput Surv 44.

2. Mendes DJM, Rodrigues IP, Baeta CF, Solano-Rodriguez C (2015) Extended clinical discourse representation structure for controlled natural language clinical decision support systems. Int J Rel Qua E-Health 4: 1-11.

3. Kazakov Y, Krötzsch MR, Simančík F (2013) The incredible ELK. J Aut Rea 53: 1-61.

4. Roberts A, Gaizauskas R, Hepple M, Demetriou G, Guo Y, et al. (2009) Building a semantically annotated corpus of clinical texts. J Biomed Inf 42: 950-966.

5. Atzori L, lera A, Morabito G (2010) The Internet of Things: A survey. Compu Net 54: 2787-2805

6. Memon M, Wagner SR, Pedersen CF, Beevi FHA, Hansen FO (2014) Ambient assisted living healthcare frameworks, platforms, standards, and quality attributes. Sensor 14: 4312-4341.

7. Ylisaukko-oja A, Vildjiounatie E, Mantyjarvi J (2004) Five-point acceleration sensing wireless body area network-design and practical experiences. Int Sym Wear Comput 1:184-185

8. Bae J, Cho H, Song K, Lee H, Yoo HJ (2012) The signal transmission mechanism on the surface of human body for body channel communication. IEEE 60: 582-593.

9. Wegmüller MS (2007) Intra-body communication for biomedical sensor networks. ETH.

10. Yoo HJ, Bae J (2013) Low energy wireless body area network systems. In Wireless Symposium (IWS), IEEE 2013: 1-2

11. Vallejo M, Recas J, Valle D, Arc PG, Ayala JL (2013) Accurate human tissue characterization for energy-efficient wireless on-body communications. Sensor 13: $7546-7569$
12. World Health Organization (WHO) (2001) The international classification of functioning, Dis Healt 18: 237.

13. Loader BD, Hardey M, Keeble L (2008) Health informatics for older people: A review of ICT facilitated integrated care for older people. Int J Soc Welf 17 46-53.

14. Cocosila M, Archer N (2010) Adoption of mobile ICT for health promotion: An empirical investigation. Elect Mark 20: 241-250.

15. Haux R, Howe J, Marschollek M, Plischke M, Wolf K (2008) Health-enabling technologies for pervasive health care: on services and ICT architecture paradigms. Inf Healt Soc Care 33: 77-89.

16. European Union (2010) ICT \& Ageing-European study on users, markets and technologies. European Commission.

17. Erden F, Velipasalar S, Alkar AZ, Cetin AE (2016) Sensors in assisted living

18. Heart T, Kalderon E (2013) Older adults: Are they ready to adopt health-related ICT? Int J Med Inf 82: 209-231.

19. Kozar-Westman M, Troutman-Jordan M, Nies MA (2013) Successful aging among assisted living community older adults. J Nurs Sch 45: 238-246.

20. Li R, Lu B, McDonald-Maier KD (2015) Cognitive assisted living ambient system: A survey. Dig Commu Net 1: 229-252.

21. Mowafey S, Gardner S (2013) Towards ambient intelligence in assisted living: The creation of an Intelligent Home Care. Sci Inf Conf 1: 51-60.

22. Rashidi P, Mihailidis A (2013) A survey on ambient-assisted living tools for older adults. IEEE J Biomed Healt Inf 17: 579-590.

23. Fillmore H, DuBard CA, Ritter GA, Jackson CT (2014) Health care savings with the patient-centred medical home: community care of North Carolina's experience. Popu Healt Manage 17: 141-148. 